\title{
Left ventricular strain analysis by positron emission tomography: Beyond myocardial perfusion
}

\author{
Letizia Spinelli, $M D^{a}$ \\ a Department of Advanced Biomedical Sciences, University of Naples Federico II, Naples, Italy
}

Received Jul 3, 2021; accepted Jul 7, 2021

doi: $10.1007 / \mathrm{s} 12350-021-02747-y$

\section{See related article, pp. 2103-2114}

In the recent years, there has been a clear shift toward increased utilization of cardiac positron emission tomography (PET) in clinical use for the assessment of myocardial perfusion imaging. ${ }^{1-3}$ The information provided by cardiovascular PET imaging is complementary to the anatomic and functional evaluation by computed tomography, cardiovascular magnetic resonance (CMR), and echocardiography. Quantitation of myocardial blood flow (MBF) and myocardial flow reserve (MFR) with the use of PET confers advantages over other modalities related to the functional evaluation of the severity of coronary stenosis. Indeed, PET imaging allows the detection of myocardial ischemia with a superior diagnostic accuracy (accurate scatter and attenuation correction) and a lower patient radiation exposure than single-photon emission computed tomography. Nitrogen-13-ammonia (13 N-ammonia) PET has been widely used for quantification of MBF in scarred and non-infarct-related areas and to assess microvascular dysfunction in asymptomatic subjects with cardiovascular risk factors and in patients with coronary artery disease or other cardiac disorders. ${ }^{2,3}$

Advanced analysis of myocardial imaging enables accurate measurement of local frame-to-frame displacement and velocity (ratio between displacement and time interval). Such methods are commonly referred as speckle tracking in echocardiography, and as feature

Reprint requests: Letizia Spinelli, MD, Department of Advanced Biomedical Sciences, University of Naples Federico II, Via Pansini 5, 80131 Naples, Italy; letspine@unina.it

J Nucl Cardiol 2022;29:2115-8.

1071-3581/\$34.00

Copyright (C) 2021 American Society of Nuclear Cardiology. tracking or border tracking when applying to other image modality such as CMR. Feature tracking is a technology that can lead to more automation and robustness of quantitative analysis of medical images with less time consumption than comparable methods. Feature tracking applied to CMR has been validated versus myocardial tagging with harmonic phase imaging and speckle tracking echocardiography. ${ }^{4,5}$ Given the complex architectural arrangement of myofibers in circumferential and longitudinal directions, measurements of myocardial deformation (strain) are performed along three principal vectors that describe left ventricular (LV) mechanics. Thus, circumferential strain, longitudinal strain and radial strain are utilized for the detecting subtle changes of global and regional LV function in many clinical situations.

In this issue of the Journal, Kawakubo et al report on the application of a feature tracking algorithm, which has been previously developed for myocardial strain analysis in CMR, to electrocardiogram-gated high-resolution cine imaging by $13 \mathrm{~N}$-ammonia PET to obtain a noninvasive absolute quantification of LV mechanics and of MBF on a single data scan and with a single tracer. ${ }^{6}$ They retrospectively analyzed PET cine imaging from 95 patients with greater than $50 \%$ stenosis of at least one coronary artery and ten healthy subjects to explore the changes in regional and global LV function during adenosine stress test, along with myocardial perfusion and MFR assessment. Findings shed light on a different behavior of patients compared to control group. At rest, the values of longitudinal strain were lower in patients than in controls, while circumferential strain values were similar. During adenosine stress test, either longitudinal or circumferential strain increased in control group. Conversely, compared to resting values, circumferential strain significantly decreased in patients with $\mathrm{MFR}<2$, while was unchanged in patients with MFR $>2$. Longitudinal strain did not change in neither of patient subgroups. This finding should not be 
interpreted as a failure of longitudinal strain in detecting myocardial ischemia. In the animal model, assessment of circumferential and longitudinal strain is crucial to the quantitative evaluation of $\mathrm{LV}$ deformation during acute myocardial ischemia. ${ }^{7}$ However, the acute induction of a coronary artery constriction is not truly representative of what happens in humans, who experience chronic progression of coronary artery disease. Reduced resting values of longitudinal strain may be found in coronary artery disease patients, since subendocardial myocardial fibers, which are mainly longitudinally oriented, are more susceptible to ischemia and repetitive stunning. In such a case no further decline in longitudinal strain may be observed during stress testing. Conversely, changes in circumferential strain with adenosine stress well reflect the ischemia-induced dysfunction of middle myocardial wall, where myocardial fibers are circumferentially oriented. Thus, the transmural degree of contractile impairment in ischemic myocardium can be evaluated by the combined use of longitudinal and circumferential strain. Adding deformation analysis to myocardial perfusion and MFR evaluation could be a valuable tool for achieving a more precise comprehension of ischemia-related myocardial dysfunction. This is all the more true since no changes in the LV ejection fraction values are often observed during stress induced ischemia in coronary artery disease patients. The authors should be lauded for exploring the implementation of an already clinically available resource to improve the diagnostic pathways. With the rapid development of new image analysis techniques, the potential to significantly improve our understanding of imaging biomarkers is meaningful. Current findings need to be tested and validated in large and potentially multicenter studies. This implies that analysis algorithms should be widely available. Further research is warranted to address the reliability of deformation parameters by PET imaging in evaluating severity of coronary artery disease, guiding management of myocardial ischemia, and differentiating microvascular disease from flow-limiting epicardial coronary artery stenosis. Notably, not only reduction in peak systolic strain, but also systolic lengthening and post-systolic shortening may be characteristic features of ischemic dysfunction. These concepts should be kept in mind while applying strain imaging to diagnose myocardial ischemia. The impairment in systolic function may be missed if peak strain is considered instead of end-systolic strain, since marked post-systolic shortening may result in near normal peak strain. Hence, it is important to take timing into account when reporting values for peak strain from ischemic myocardial segments.

One might wonder whether adding deformation analysis to PET would facilitate the diagnosis of viable myocardium in patients with ischemic cardiomyopathy. Fluorine 18 deoxyglucose (18F-FDG) PET imaging is considered the gold standard for identifying myocardial viability and is often used to guide surgical or percutaneous interventional management. ${ }^{8}$ Absolute quantification of resting $\mathrm{MBF}$ with the use of $13 \mathrm{~N}$ ammonia PET has proved to be valuable in distinguishing hibernating and stunned viable myocardium from infarcted myocardium and to provide complementary practical value to regional myocardial wall thickening as assessed by CMR. ${ }^{9,10}$ Combining peak systolic longitudinal strain with the additional information relative to post-systolic deformation provides the most informative deformation parameters to predict the transmural extent of necrosis in the early and late phases of myocardial infarction. It is worth to note that postsystolic strain, which is generally considered a marker of viability, may occur also in myocardium with transmural necrosis or scar. Nevertheless, in the latter case there is typically a systolic lengthening with post-systolic shortening, due to passive recoil during isovolumic relaxation. Hence, data provided by strain imaging need to be carefully interpreted. The potential value of adding myocardial deformation analysis (both peak systolic strain and time to peak systolic strain) to gated PET imaging for the evaluation of myocardial viability deserves to be investigated.

Additionally, a potential role for myocardial deformation analysis by PET in risk stratification of patients with ischemic cardiomyopathy could be hypothesized. Myocardial perfusion findings by PET have an incremental prognostic value over routine clinical predictors for all-cause mortality and major adverse cardiac events in patients with known or suspected coronary artery disease. A warranty period of 3 years has been demonstrated if normal MFR is associated with normal perfusion. Conversely, in patients with abnormal perfusion, an impaired MFR has added value. ${ }^{11}$ Conceivably, strain analysis might reveal a prognostic impact over perfusion information for optimized risk stratification following acute myocardial infarction. After adjustment for traditional cardiac risk factors, global longitudinal strain by CMR was the strongest predictor of adverse prognosis in a large highrisk population recovering from acute myocardial infarction, with an incremental prognostic value in addition to $\mathrm{LV}$ ejection fraction and infarct size for the prediction of all-cause mortality. ${ }^{12}$

Beyond myocardial viability, cardiac metabolism study with the use of 18F-FDG PET has already gained applications in non-ischemic cardiomyopathy. Myocardial damage related to cardiac sarcoidosis or other cardiomyopathies is becoming an increasingly important field of investigation for PET imaging. The combined 
assessment of perfusion and inflammation has proven to provide additional information about the status of cardiac sarcoidosis and is the currently recommended radionuclide method for evaluation of this disease. ${ }^{13}$ Adding strain analysis to metabolic and perfusion data by PET would provide a diagnostic tool to define the relationships between disease activity and changes in $\mathrm{LV}$ function on the base of a segmental and global analysis and to identify sub-clinical LV dysfunction in patients with preserved or normal LV ejection fraction. Indeed, both speckle tracking echocardiography and strain CMR indicate that global longitudinal strain may serve as a marker for early cardiac involvement in sarcoidosis, due the fact that it is the only parameter detecting LV functional impairment in sarcoidosis patients with otherwise unsuspicious cardiac disease. Importantly, there is evidence that global longitudinal strain is also independently associated with occurrence of adverse events. ${ }^{14,15}$ In Anderson-Fabry disease, cardiac 18F-FDG PET detects myocardial inflammation that likely represents a clue of response to cardiomyocyte globotriaosylceramide storage. We performed a head to-head comparison of hybrid cardiac PET and CMR and 2-dimensional strain echocardiography in female carriers of pathogenic mutations, hence, we could demonstrate that abnormal 18F-FDG uptake was associated to impaired LV longitudinal systolic strain. ${ }^{16}$ Strain imaging by cardiac PET would have the potential to provide evidence regarding not only presence and grade of inflammation but also myocardial function impairment.

The study of Kavakubo et $\mathrm{al}^{6}$ demonstrating the possibility to clinically assess blood flow and quantify endocardial motility in ischemia patients using only a single examination, might open a new perspective in cardiac imaging by radionuclide techniques. This novel imaging tool could apply to many clinical situations where, alongside with perfusion and metabolism assessment, global and regional LV function analysis is meaningful. However, prospective clinical trials would be required to investigate the added clinical value that strain by cardiac PET may represent in patient management.

\section{Disclosure}

The authors have indicated that they have no financial conflict of interest.

\section{References}

1. Schindler TH, Schelbert HR, Quercioli A, Dilsizian V. Cardiac PET imaging for the detection and monitoring of coronary artery disease and microvascular health. JACC Cardiovasc Imaging 2010;3:623-40.
2. Nkoulou RN, et al. Diagnostic value of $13 \mathrm{~N}$-ammonia myocardial perfusion PET: Added value of myocardial flow reserve. J NuclMed 2012;53:1230-4.

3. Berti V, Sciagrà R, Neglia D, Pietilä $M$, Scholte AJ, Nekolla $S$, et al. Segmental quantitative myocardial perfusion with PET for the detection of significant coronary artery disease in patients with stable angina. Eur J Nucl Med Mol Imaging 2016;43:1522-9.

4. Hor KN, Gottliebson WM, Carson C, Wash E, Cnota J, Fleck R, et al. Comparison of magnetic resonance feature tracking for strain calculation with harmonic phase imaging analysis. JACC Cardiovasc Imaging 2010;3:144-51.

5. Onishi T, Saha SK, Delgado-Montero A, Ludwig DR, Onishi T, Schelbert EB, et al. Global longitudinal strain and global circumferential strain by speckle-tracking echocardiography and feature-tracking cardiac magnetic resonance imaging: Comparison with left ventricular ejection fraction. J Am Soc Echocardiogr 2015;28:587-96.

6. Kawakubo M, Nagao M, Yamamoto A, Nakao R, Matsuo Y, Kenji S, Watanabe E, Sakai A, Sasaki M, Sakai, Shuji 13N-ammonia positron emission tomography-derived endocardial strain for the assessment of ischemia using feature-tracking in high-resolution cine imaging. J Nucl Cardiol.

7. Suhail MS, Wilson MW, Hetts SW, Saeed M. Magnetic resonance imaging characterization of circumferential and longitudinal strain under various coronary interventions in swine. World J Radiol 2013;5:472-83.

8. Nienaber CA, Brunken RC, Sherman CT, Yeatman LA, Gambhir SS, Krivokapich J, et al. Metabolic and functional recovery of ischemic human myocardium after coronary angioplasty. J Am Coll Cardiol 1991;18:966-78.

9. Wang J, Li JM, Li S, Hsu B. Absolute Resting 13 N-ammonia PET myocardial blood flow for predicting myocardial viability and recovery of ventricular function after coronary artery bypass grafting. J Nucl Cardiol. 2020. Epub ahead of print. PMID: 33089879.

10. Tawakol A, Skopicki HA, Abraham SA, Alpert NM, Fischman AJ, Picard MH, et al. Evidence of reduce resting blood flow in viable myocardial regions with chronic asynergy. J Am Coll Cardiol 2000;36:2146-53.

11. Herzog BA, Husmann L, Valenta I, Gaemperli O, Siegrist PT, Tay $\mathrm{FM}$, et al. Long-term prognostic value of $13 \mathrm{~N}$-ammonia myocardial perfusion positron emission tomography added value of coronary flow reserve. J Am Coll Cardiol 2009;54:150-6.

12. Eitel I, Stiermaier T, Lange T, Rommel KP, Koschalka A, Kowallick JT, et al. Cardiac magnetic resonance myocardial feature tracking for optimized prediction of cardiovascular events following myocardial infarction. JACC Cardiovasc Imaging 2018;11:1433-44.

13. Slart RHJA, Glaudemans AWJM, Lancellotti P, Hyafil F, Blankstein R, Schwartz RG, et al. A joint procedural position statement on imaging in cardiac sarcoidosis: From the Cardiovascular and Inflammation \& Infection Committees of the European Association of Nuclear Medicine, the European Association of Cardiovascular Imaging, and the American Society of Nuclear Cardiology. J Nucl Cardiol 2018;25:298-319.

14. Joyce E, Ninaber MK, Katsanos S, Debonnaire P, Kamperidis V, Bax JJ, et al. Subclinical left ventricular dysfunction by echocardiographic speckle-tracking strain analysis relates to outcome in sarcoidosis. Eur J Heart Fail 2015;17:51-62.

15. Dabir D, Meyer D, Kuetting D, Luetkens J, Homsi R, Pizarro C, et al. Diagnostic value of cardiac magnetic resonance strain analysis for detection of cardiac sarcoidosis. Rofo 2018;190:71221. 
16. Spinelli L, Imbriaco M, Nappi C, Nicolai E, Giugliano G, Ponsiglione A, et al. Early cardiac involvement affects left ventricular longitudinal function in females carrying $\alpha$-galactosidase a mutation: Role of hybrid positron emission tomography and magnetic resonance imaging and speckle-tracking echocardiography. Circ Cardiovasc Imaging. 2018;11:
Publisher's Note Springer Nature remains neutral with regard to jurisdictional claims in published maps and institutional affiliations. 